\title{
Porter plainte en justice
}

Dynamique des prises de parole de la dispute à l'action en justice

\section{Mickaële Lantin Mallet}

\section{(2) OpenEdition}

Journals

Édition électronique

URL : https://journals.openedition.org/clo/2361

DOI : $10.4000 /$ clo.2361

ISSN : 2266-1816

Éditeur

INALCO

Édition imprimée

Date de publication : 31 décembre 2015

ISBN : 9782858312276

ISSN : 0396-891X

Référence électronique

Mickaële Lantin Mallet, « Porter plainte en justice », Cahiers de littérature orale [En ligne], 77-78 | 2015, mis en ligne le 10 mai 2016, consulté le 30 juin 2021. URL : http://journals.openedition.org/clo/2361 ; DOI : https://doi.org/10.4000/clo.2361

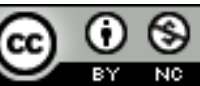

Cahiers de littérature orale est mis à disposition selon les termes de la Licence Creative Commons Attribution - Pas d'Utilisation Commerciale 4.0 International. 


\title{
Porter plainte en justice Dynamique des prises de parole de la dispute à l'action en justice
}

\author{
Mickaële Lantin Mallet \\ Iris, EHESS
}

Dans le cadre des conflits entre proches, la parole constitue souvent la matière première à partir de laquelle se développent les tensions et éclatent les disputes : qui a dit quoi et à qui, de quelle manière et quand, pourquoi et de quel droit ? Les récits de conflits que nous avons pu recueillir auprès de personnes ayant porté plainte en justice à la suite d'un différend avec des proches comprennent régulièrement des références à des actes de langage perçus par le plaignant comme des offenses ayant initié ou participé à la dynamique de la dispute : paroles qualifiées de provocatrices, d'insultantes ou de mensongères, engagements verbaux rompus, confidences divulguées, mais aussi absence de dialogue ou de réponse dans le cadre de salutations, etc.

L'échange verbal et la parole sont enfin également constitués par les parties en lice lors de différends, ainsi que par l'institution judiciaire, comme un des moyens par lequel le différend pourrait s'apaiser ou se résoudre. Ainsi, certains disputants choisissent de porter plainte pour modifier ou faire cesser les échanges conflictuels. Les plaignants signalent, dans le corps de leur plainte en justice, des actes remédiateurs aux offenses qu'ils estiment avoir subies comme, par exemple, l'acceptation, par la personne incriminée, à reprendre le dialogue, à livrer des explications quant à sa conduite, à faire des excuses, à prendre part à une réunion familiale ou à un rendez-vous médical, notarial, etc. L'institution judiciaire française dispose, pour répondre aux actions en justice, de différents dispositifs de gestion des conflits et litiges, organisés par et autour des prises de parole des parties litigantes, de leurs représentants, des magistrats et, parfois, d'autres classes d'intervenants (témoins, experts juridiques, médiateurs et conciliateurs, etc.). L'activation de ces dispositifs judiciaires requiert le plus souvent émission 
d'une plainte, enregistrement de celle-ci par un interlocuteur ad hoc (gendarme, policier, procureur) et, enfin, orientation de l'affaire ainsi constituée vers une instance spécialisée qui procédera à son traitement (médiation pénale, rappel à la loi, audience de comparution immédiate, etc.). Le dispositif sur lequel nous avons travaillé en Martinique, la médiation pénale, participe d'une politique judiciaire dite de la justice de proximité mise en œuvre en France dès le début des années 1990 et étendue progressivement au territoire hexagonal et aux départements d'outre-mer. Les codes, règles et procédures encadrant ce mode de résolution des litiges ne sont en rien spécifiques à chaque territoire juridictionnel, cependant leur mise en pratique relève de stratégies singulières propres aux divers acteurs impliqués à l'échelon local ${ }^{1}$, à leurs relations préexistantes ainsi qu'aux spécificités socioculturelles et historiques locales (rapport au droit et aux institutions marqué par le passé colonial, notamment pour la Martinique).

Une plainte en justice déclenche, lorsqu'elle est estimée recevable pénalement, une action publique au travers de laquelle l'institution judiciaire entend poursuivre et punir le ou les auteur(s) des faits signalés. Par là même, les actions engagées par le plaignant, sa plainte, sont reconnues comme justiciables.

Dans le cadre de cet article, nous nous attacherons à retracer le chemin de la plainte en justice en remontant le courant : les récits produits par les plaignants à l'occasion de leur action en justice permettent de retracer les parcours réalisés par ceux-ci pour faire reconnaître leur problème dans l'espace judiciaire. Ces parcours de plaignants seront documentés à partir des récits ${ }^{2}$ produits dans

1. La mise en place d'une maison de justice et du droit offrant accès aux services de médiation pénale suppose en effet l'implication des agents locaux de l'institution judiciaire (notamment le procureur et le barreau local), des associations habilitées par l'institution judiciaire à effectuer des missions socio-judiciaires sur mandat (telles que l'aide aux victimes, le suivi socio-éducatif et juridique, la réparation pénale, etc.), mais aussi des responsables locaux de la politique de la ville. Lors de nos deux terrains en Martinique, les litiges orientés de manière privilégiée vers la médiation pénale impliquaient tous des parties ayant entre elles des relations interpersonnelles supposant un degré élevé de proximité géographique et physique sur la longue durée (relation conjugale, familiale ou vicinale). D'autre part, les médiateurs pénaux en fonction provenaient alors tous du secteur de l'éducation spécialisée et appartenaient à une association partenaire de l'institution judiciaire depuis les années 1980, spécialisée dans le suivi socio-éducatif et judiciaire et les enquêtes de personnalité. Cette association fut également celle qui a obtenu la gérance de la maison de justice et du droit dès sa création en 1999.

2. Dans le corps de l'article et dans les citations, les récits sont identifiés par deux lettres : LP pour lettre de plainte, MP pour médiation pénale ; suivies d'un numéro correspondant à leur catégorie et place au sein de nos différents corpus de données ethnographiques. 
différents contextes : dans le cadre de lettres de plainte adressées au procureur de la République de la Martinique, lors des entretiens ethnographiques avec des personnes ayant connu un épisode judiciaire (médiation pénale) et, enfin, lors des sessions de médiation pénale au sein de la maison de justice et du droit de Fort-de-France. Dans un second temps, seront analysés les risques attachés à l'émission d'une plainte en justice dans les contextes étudiés (Martinique, conflits interpersonnels entre proches) et, enfin, on examinera les stratégies rhétoriques mises en œuvre par les plaignants que ce soit pour déjouer les risques associés à l'acte de porter plainte en justice ou pour s'assurer de la bonne réception de leur plainte, ici par le procureur de la République.

\section{Des récits de malheur à la plainte en justice}

Dans le cadre d'un différend interpersonnel, la plainte est l'acte par lequel un énonciateur dit « plaignant » adresse à un récipiendaire un énoncé qui constitue tout à la fois :

- L'expression d'un état émotionnel (mécontentement, détresse, indignation, etc.).

- Le signalement d'un problème souvent accompagné d'imputations causales (raisons et auteur(s) dudit problème). Ce signalement est souvent réalisé sous la forme d'un récit exposant l'histoire du différend, les éléments constitutifs de la situation problématique et un travail de portraiture des personnes impliquées.

- Une demande d'intervention visant à assurer le règlement du problème et l'administration de sanctions au(x) auteur(s) de celui-ci.

Les moyens par lesquels est formulée cette demande délivrent au récipiendaire de la plainte, de manière plus ou moins explicite, des indications quant aux types d'intervention attendus du solliciteur pour régler la situation problématique ou ses conséquences (nature de l'intervention, des sanctions, etc.). La réalisation d'une plainte en justice requiert plus particulièrement un repérage des interlocuteurs pertinents auxquels adresser sa plainte, un travail de compréhension et de description de faits susceptibles de constituer une infraction ${ }^{3}$. L'auteur d'une plainte en justice est animé par de multiples attentes envers l'institution judiciaire qui concernent la légitimation de son action en justice, la reconnaissance des procédés résolutoires ou punitifs qu'il indique ainsi que la mise en œuvre de

3. Une infraction est un comportement ou une action condamnée et sanctionnée par la loi. En fonction des comportements ou actions considérés on parlera, en droit français, de contravention, de délit et de crime. 
CAHIERS DE LITTÉRATURE ORALE

146 Paroles publiques, paroles confidentielles $-\mathrm{n}^{\circ} 77-78$

moyens adaptés et efficaces pour assurer sa mise en sécurité et restaurer sa « tranquillité ${ }^{4}$.

Le moment de la plainte en justice échappe souvent à l'ethnographe, car la majorité des appels à l'institution judiciaire se déroulent en contexte domestique, dans le cours de disputes et souvent selon une temporalité qui est celle de l'urgence. L'ethnographe arrive bien souvent après ces « scènes » de primoplainte en justice lorsqu'il ne s'agit, pour le plaignant, que de formaliser sa plainte en l'enregistrant en contexte institutionnel (gendarmerie, commissariat). Toutefois, dans le cadre des médiations pénales, les auteurs des plaintes en justice reviennent sur la scène initiale de la plainte : comment, sur un plan pratique, et dans quelles conditions ils ont interpellé l'institution judiciaire. Ces récits permettent donc, rétrospectivement, de saisir comment se déclenche la plainte en justice. Les extraits $^{5}$ ci-dessous ( 1 et 2 ) sont issus des prises de parole de plaignants dans le cadre de premières rencontres de médiation pénale. Ils reviennent sur le moment et les circonstances dans lesquels la décision de recourir à l'action en justice s'insère. En l'occurrence, l'un et l'autre ont procédé à un appel téléphonique. Le dernier extrait (3) concerne une autre temporalité et modalité du dépôt de plainte, en l'occurrence, par la rédaction d'une lettre de plainte au procureur de la République en différé de l'offense subie par le plaignant.

4. Les plaignants signalent une rupture de l'ordinaire : « le trouble de ma tranquillité » (LP20), «il ne m'a jamais laissée tranquille » (LP8), et ils espèrent un retour à celui-ci : « avoir ma paix » (LP7), « je voudrais ma paix » (LP9), « il va me laisser tranquille et me laisser en paix » (entretien). Un autre élément vient caractériser la description de cet état de paix et de tranquillité propre à l'ordinaire pour les plaignants : c'est le fait de ne pas se « mêler » ou de ne pas être « mêlé », au sens de «s'occuper des affaires d'autrui », thématique et expression récurrente dans les différends interpersonnels en Martinique et, plus largement, aux Antilles françaises (Bougerol, 1997, 1999, 2001).

5. Les dates, noms et lieux des extraits ont été modifiés ainsi que les éléments permettant d'identifier les personnes. L'orthographe a par contre été conservée ainsi que les caractéristiques graphiques particulières utilisées par le scripteur (majuscules, guillemets, surlignage, etc.). À quelques exceptions près, toutes les lettres de plainte collectées dans les dossiers de médiation pénale sont des lettres manuscrites rédigées sur de simples feuilles vierges. Les éléments illisibles sont signalés entre crochets. Enfin, les phrases qui apparaissent en gras dans les transcriptions de fragments d'entretien ou de lettres de plainte présentées dans cet article correspondent à nos mises en exergue et non à celles des énonciateurs. 


\section{Le contexte d'interpellation des gendarmes}

Cas 1 : MP Mme Astier Colette vs M. Belin, violences avec arme, relations de voisinage

J'entends ma mère qui dit : Colette Colette ne sort pas, ne sors, n'ouvre pas la porte. Et ce monsieur-là qui, il a avec son fusil comme ça braqué son fusil comme ça [geste iconique] j'ouvre la porte, je dis : mais qu'est-ce qui se passe, pourquoi ma mère crie comme ça ? [...] c'est de là que j'ai appelé, là je suis montée, j'ai pas appelé parce que j'avais pas de téléphone là. J'ai appelé la gendarmerie, je suis montée à la gendarmerie aussi, mais là on me connaît déjà à la gendarmerie de $X$. On voit comment je vis, tout ce que j'ai subi et tout alors ils sont venus tout de suite. Ils ont... ils ont téléphoné au procureur [...] mois je... non sincèrement j'ai trop, trop vécu. J'ai pleuré, j'ai... je suis partie, j'étais en chemise de nuit en train de nettoyer ma maison, je suis partie en chemise de nuit à la gendarmerie... j'ai pris la clé de ma voiture, je suis allée en chemise de nuit à la gendarmerie avec mes deux enfants et $j$ 'ai laissé mon autre fils dans la maison, je l'ai fermé à clé et je suis partie comme ça pour aller chercher les gendarmes parce qu'il ne s'est pas arrêté comme ça.

Cas 2 : MP M. Aiglemer vs M. Vince, dégradations de bien, menaces avec arme blanche entre voisins

M. A : Ma femme m'a dit : surtout ne descends pas, tu as à perdre plus qu'à gagner ! J'ai dit : tu as raison, laisse, laisse tomber, laisse le faire. Tout d'un coup j'entends avec son coutelas il tapait sur l'interphone. Non seulement le mien, mais aussi celui des voisins ! Quand il a vu que je venais pas il a commencé à s'attaquer à ma voiture. Le pare-brise, les quatre pneus, une jante à coups de coutelas, les deux rétroviseurs, le volet arrière, l'antenne aussi... A pas pouvoir ne pas ne pas descendre. Alors elle a dit : non, elle a dit : laisse rien, laisse tout ça c'est du matériel tout ça, laisse on va appeler les flics. On va appeler les flics on va appeler les flics ! Il ira à Ducos [centre pénitenciaire] comme ça on sera débarrassés de lui pendant un moment, laisse-le faire ! C'est ce que j'ai fait. J'ai appelé les flics. 
CAHIERS DE LITTÉRATURE ORALE

148 Paroles publiques, paroles confidentielles $-\mathrm{n}^{\circ} 77-78$

\section{Le contexte d'interpellation du procureur de la République : la lettre de plainte}

Cas 3 : LP Mme Jonahan vs M. Jonathan, menaces avec armes, coups et blessures entre conjoints

Mon mari ne m'a jamais laissée tranquille, il continue de me persécuter, il continue à faire des désordres avec moi, se battre avec moi, il est toujours violent avec les enfants [...] Excusez-moi d'avoir fait le courrier sur ce papier, c'est parce qu'il n'était pas loin avec son (coutelas ?) mis le verrou alors j'ai profiter de la faire vivement car c'est ce qui était sur mes mains ${ }^{6}$.

Ces trois « moments » de plainte montrent que les plaignants saisissent l'outil « plainte en justice » sous la pression, dans des situations où ils sont confrontés à la nécessité de prendre une décision : comment et quel type d'action poser pour faire face aux conduites d'une personne mettant en jeu leur sécurité, leur santé, leurs biens, etc. Les plaignants, parfois accompagnés de leurs proches (cas 2), évaluent la pertinence de passer à la plainte en justice par rapport à ce qu' ils escomptent en matière de conséquences immédiates (arrêt des agressions) et à long terme (sanction de l'agresseur). Les plaintes sont ainsi classifiables selon qu'elles sont de type direct ou indirect, en face-à-face ou médiées par l'écrit, informelles, institutionnelles ou officielles juridiquement (avec différents degrés dans l'officiel comme le montre la distinction entre main courante et plainte en justice sur laquelle nous reviendrons plus bas).

\section{Le parcours du plaignant : agir « avant qu'un drame ne se produise »}

Les extraits précédents s'inscrivent dans des « récits du malheur $\gg{ }^{7}$ plus larges qui décrivent des situations caractérisées par l'urgence et la détresse : le scripteur manifeste un besoin impérieux de prise au sérieux de sa parole par ceux à qui il l'adresse et que celle-ci déclenche une prise en charge de la situation qu'il expose. Les plaignants signifient clairement que cet acte - porter plainte - est la dernière manifestation verbale de leur mécontentement, leur ultime demande d'aide avant qu'ils ne passent à d'autres modes de gestion de leur problème comme, par

6. Courrier rédigé sur une feuille arrachée d'un agenda, écriture manuscrite en biais.

7. Jean-François LaÉ, Numa Murard, 1995. 
exemple, le recours à la justice privée, à une vengeance par actes physiques violents envers autrui, ou encore à des actes autodestructeurs :

Monsieur le Procureur, je suis au stade du suicide, je frappe à toutes les portes, je n'ai aucun résultat. Vous êtes mon seul appui, mon seul secours, SVP, aidez-moi ! Venez à mon secours ! Je n'en peux plus !! [...] Par ailleurs, avant de faire une dépression ou avant de faire justice moi-même, je vous préviens à chaque reprise. (LP7)

La plainte est décrite comme un acte situé à l'acmé de l'histoire du conflit : le plaignant signifie que ses « limites ont été franchies » (LP2), que ses ressources (patience, santé, autres modes de résolution du problème, etc.) ont été épuisées et signale que ce qu'il vit ressort du registre de l'anormal, de l'insupportable et de l'intolérable : « Tout ce que nous savons c'est que le quotidien est invivable » (MP9), ce que « nous subissons (...) intolérables pour des personnes normalement constituées $\gg($ LP17).

La plainte en justice est ainsi présentée et investie par les plaignants comme la seule voie possible et efficace pour résoudre leurs difficultés, mais aussi comme le moyen par lequel seront évitées l'escalade violente entre les parties en lice et la survenue possible d'un drame. Pour autant, la plainte en justice ne constitue pas le seul moment et la seule occurrence où le plaignant expose publiquement ses difficultés et sollicite l'aide de tiers concernant les relations avec des proches. L'ensemble des récits de plaignants recueillis sur le terrain fait état des itinéraires réalisés en quête de solutions à ce qui, pour eux, constitue un problème. Ils font apparaître plusieurs séquences d'émission de plaintes hors du cadre judiciaire stricto sensu. Celles-ci ont pu être adressées à des proches (personnes relevant du cercle familial, vicinal ou amical), à des figures locales assumant la fonction de conciliateur ou d'arbitre à l'échelle du quartier ou du groupe d'appartenance, de personnes dont l'activité ou la profession les désigne comme des « aidants » possibles en cas de violences interpersonnelles (avocat, assistante sociale, membres d'associations caritatives spécialisées, médiateur de quartier ou médiateur familial par exemple) ou encore à des figures religieuses ou étatiques assumant également des fonctions de régulation sociale (prêtre, pasteur, maire, policier, gendarme, etc.).

La description du parcours et la thématique de la quête sont des éléments communs repérables dans tous les récits de plaignants que nous avons recueillis, ce indépendamment des disparités des motifs de plaintes (type de problème), de la variété des relations unissant les parties en lice, des contextes et des moyens d'énonciation du récit de plaignant (lettre de plainte, médiation pénale, entretien ethnographique). Les récits rendent ainsi compte de la quête par le plaignant d'une 
personne ou d'une instance qui puisse l'écouter et intervenir, d'un interlocuteur comprenant et partageant son point de vue sur ce que sont ses motifs de plainte et d'indignation.

Identifier celui qui occupe la position de réception ou d'évaluation de la plainte permet de comprendre comment leur auteur procède à des réajustements progressifs et successifs dans la formulation de sa plainte lorsqu'il est amené, comme dans les cas étudiés, à réémettre celle-ci en direction du même récipiendaire ou d'autres interlocuteurs. En fonction de la personne, ou de l'instance à qui la plainte est adressée et selon le sort qu'auront connu ses plaintes antérieures, le plaignant retravaille la présentation de son « problème », reformule et réagence les éléments qui constituent pour lui les clés de compréhension de son histoire et de sa situation conflictuelle présente. Par ailleurs et à chaque nouvelle plainte, l'auteur de celles-ci relance et réoriente sa quête d'interlocuteurs susceptibles de satisfaire ses demandes, interprète les raisons de son insuccès à se faire entendre et s'appuie sur l'ensemble de son parcours de plainte et d'expériences de prise de parole pour s'engager dans une nouvelle séquence d'action.

La plainte en justice, non plus en tant qu'acte de prise de parole en soi, mais en tant que récit et mise en intrigue d'un événement, est donc le produit d'une constellation de récits antérieurs. Au fur et à mesure que le conflit évolue, le plaignant sollicite divers interlocuteurs et leur formule ce qu'est sa situation et ses attentes. En réponse il obtient certes écoute, conseils et orientations, mais surtout un travail de traduction de ses expériences, de reformulation de ses propos, de requalification de son vécu. Et si ses interlocuteurs peinent à résoudre tout ou partie des difficultés que le plaignant estime endurer, ils le confrontent par leur mode de réception de sa plainte - indifférence, doute, contestation, rejet, mais aussi questions, conseils, etc. - à la nécessité de produire un récit plus détaillé ou factuel qu'il ne l'était initialement (dates, présence de tiers, etc.) ; à la nécessité de fonder ses allégations et requêtes sur des preuves qu'il n'avait pas pensé à collecter (témoignages, pétition, factures, certificats médicaux) ou encore d'user de registres argumentatifs différents de ceux qu'il mobilisait spontanément (références juridiques, etc.).

Durant ce parcours, s'opère donc un travail de qualification du problème, de négociation de ce qui, de l'expérience du plaignant, peut être exposé et en quels termes et, à l'inverse, de ce qu'il est préférable de taire pour favoriser la bonne réception de sa plainte selon l'interlocuteur auquel celle-ci s'adresse ou selon la finalité que poursuit le recours à l'institution judiciaire (Fassin, 2000 ; Retières, 2001 ; Laé, Astier, 2007). Robert Emerson et Sheldon Messinger (1977, p. 123) rappellent que : 
Many troubles, particularly when first noted, appear vague to those concerned. But as steps are taken to remedy or manage that trouble, the trouble itself become progressively clarified and specified. In this sense the natural history of a trouble is intimately tied to -and produces- the effort to do something about it.

Ainsi, les termes dans lesquels les plaignants formulent initialement leur problème sont parfois, dans un second temps, amendés en raison des suites judiciaires potentielles, pour eux ou pour autrui, des signalements effectués :

Et quand je me suis rendu compte lors de l'interrogation à la gendarmerie que c'était un cas d'assises, que je ne voulais quand même pas que mon mari aille aux assises donc c'est de là que j'ai pas fait une déclaration de viol et j'ai porté plainte pour les coups et blessures par contre. (MP9)

Au-delà de ces mises en intrigue différentielles du problème en fonction des interlocuteurs auxquels s'adresse le plaignant tout au long de son parcours, retenons que la plainte en justice intervient à la suite de multiples séquences d'actions et tentatives de redressement d'une situation estimée problématique par le requérant. L'action en justice s'inscrit donc dans une généalogie de prises de parole sur le conflit prenant place au sein d'une quête d'instances disposées à prendre en charge efficacement le problème qui leur est signalé par le plaignant. Elle ne constitue donc pas un acte de publicisation du problème en soi, mais une forme parmi d'autres de mise en visibilité d'un problème qui, d'une prise de parole à l'autre et d'un contexte énonciatif à l'autre, est reformulé, voire transformé. Le conflit a donc déjà été l'objet d'échanges dans le cercle des proches et au-delà. Il a même parfois été l'objet d'interpellations d'autres représentants de l'État dans l'espace public ${ }^{8}$ au moment où le plaignant saisit l'institution judiciaire.

\section{La plainte : la fixation d'une parole publique}

Ce que « fait » la plainte en justice et que ne font pas les plaintes antérieures, c'est donner un caractère officiel à un différend qui a déjà connu une « vie publique ». En cela, elle n'est pas une prise de parole qui rend visible ce qui jusqu'alors ressortirait de l'enclos, du privé, de l'intime ou du non-dit. Par contre, l'action

8. En particulier le maire et les services municipaux (cadastre, voirie, état civil) ou des organismes publics comme la direction départementale de l'équipement, la direction régionale des affaires sanitaires et sociales. 
en justice vient inscrire, de manière officielle et stable, la parole du plaignant dans l'espace public. Ainsi, à la suite d'un dépôt de plainte, en gendarmerie, au commissariat ou par lettre au procureur, un document écrit sera établi et signé par un représentant de l'institution et par le plaignant'.

Ce que fait également la plainte, au-delà d'officialiser et de fixer les termes définissant le « problème »- le conflit est requalifié pour correspondre à des catégories et barèmes juridiques préétablis - c'est qu'elle redistribue et transforme les ressources dont les plaignants peuvent disposer pour décrire et qualifier leurs expériences et les événements ayant trait au différend. Les époux Valade du cas que nous venons d'exposer deviennent pour l'un « plaignant » et pour l'autre « mis en cause » dans un différend devenu « litige » pour « menaces de mort ». Ce litige sera désormais traité avec un tiers, le médiateur pénal et, s'il s'avère impossible d'arriver à un accord à l'amiable, les parties en présence seront renvoyées vers le tribunal et conviées à se faire représenter par un avocat.

\section{La plainte en justice : une prise de parole risquée}

Le processus de la plainte comprend plusieurs opérations qui, toutes, sont nécessaires à la constitution de cet acte, mais qui sont également toutes porteuses de risques et synonymes de difficultés à comprendre et à surmonter si le plaignant veut que son « chemin »de plainte se poursuive, que celle-ci soit émise, reçue et traitée et, corollaire heureux, que la situation justifiant sa plainte soit prise en charge et, dans le meilleur des cas, résolue.

\section{L'épreuve du silence et de l'endurance}

Les récits des plaignants montrent que faire appel à un tiers en contexte conflictuel est une pratique régulière, mais qui n'a pas toujours de conséquences directes et efficaces sur la dynamique conflictuelle. Le dépôt d'une plainte lui-même n'engage pas toujours un retour et, bien souvent, pas un retour immédiat. Le temps de traitement judiciaire de la plainte en justice autant que les possibilités que celle-ci soit « classée sans suite », sans que le plaignant en soit toujours informé, installent donc les auteurs des plaintes dans un état d'attente et d'incertitude. Ce,

9. La déclaration de plainte en gendarmerie ou commissariat n'est pas une interaction sur laquelle nous avons travaillé même si nous avons eu accès, par les dossiers judiciaires, aux procès-verbaux des affaires qui ont été traitées en médiation pénale. Nous renvoyons, pour un travail interactionnel sur le « moment » de constitution de ces procès-verbaux, aux travaux de Michel de Fornel (1988) et d’Esther González Martínez (2003). 
alors même que ceux-ci perçoivent leur situation comme critique et difficilement supportable, et qu'ils ont souvent déjà été confrontés à des absences de réponse suite à d'autres sollicitations.

Sans retour de la part de l'institution, les plaignants procèdent à une multiplication de plaintes : diverses instances sont mobilisées en simultané ou successivement, des relances sont réalisées directement en brigade de gendarmerie ou par un nouvel écrit au procureur de la République, des pièces à charge contre leur offenseur sont accumulées de manière à appuyer ce qui devient, progressivement, leur « dossier » préparatoire à la plainte ou à ses suites. Dans leurs récits les plaignants se dépeignent ainsi comme ayant produit de nombreux efforts, comme ayant manifesté beaucoup de patience et d'endurance pour porter leur plainte jusqu'à ce que celle-ci soit enfin entendue, reçue et enregistrée par l'un de leurs interlocuteurs et, dans les cas qui nous occupent, par l'institution judiciaire.

On a été à maintes reprises chez les gendarmes ils sont venus on a été chez les policiers à force de porter plainte et que ça change pas j'étais obligé ce jour-là de m'exciter pour que pour l'impressionner on n'arrive pas à déplacer les gendarmes et quand ils viennent y a pas de résultat. (MP11)

\section{Au risque de la disqualification judiciaire et sociale}

La plainte en justice constitue un « acte d'institution », c'est-à-dire que la réalisation de cet acte « est inséparable de l'existence d'une institution définissant les conditions (en matière d'agent, de lieu ou de moment, etc.) qui doivent être remplies pour que la magie des mots puisse opérer $\gg^{10}$. Si l'énonciateur de la plainte ne sacrifie pas aux actes attendus de lui pour occuper légitimement, aux yeux du droit, la place du plaignant, il encourt la disqualification de son intention d'agir en justice (réorientation du plaignant vers une tierce instance, classement sans suite). Parmi les attentes normatives auxquelles le plaignant doit répondre pour établir une plainte juridiquement valide, on trouve, par exemple, le fait de savoir repérer dans l'éventail des personnes ressources disponibles localement quels sont les interlocuteurs compétents et habilités à recevoir une plainte en justice, mais aussi le fait de distinguer l'acte de consignation d'une main courante en gendarmerie et l'acte de déclaration d'une plainte auprès de la même instance.

Les récits des plaignants manifestent des déficits d'information quant à l'aspect procédural de l'acte de porter plainte, mais aussi des difficultés de compréhension

10. Pierre Bourdieu, 1982, p. 69. 
des stratégies de qualification et de différentiation des actes susceptibles de devenir des objets pénalement valides. L'énoncé ci-après issu d'un entretien ethnographique illustre le processus tâtonnant de construction du problème rencontré par le plaignant en un fait juridiquement recevable :

[...] j'ai été, je me suis déplacée en gendarmerie. À la gendarmerie j'ai encore eu droit à un autre discours, que normalement ce n'était pas à la gendarmerie de gérer ça que c'était à la mairie puisque c'était entre deux employés [municipaux] ensuite que comme ce sont des insultes qui m'avaient été faites, entre deux personnes qu'il n'y avait rien à voir [inaudible/incompréhensible]. Donc ça m'a un peu énervé parce que, j'ai dit ça aux gendarmes, j'aurai été violée dans l'intimité cela n'aurait rien eu à voir non plus donc pour moi c'est pareil, c'est moral. Et je ne veux pas qu'on atteigne à ma vie privée comme ça. Donc j'ai déposé une première plainte. [Entretien Mme Tivoli, plaignante dans un litige traité en médiation pénale, conflit entre collègues de bureau au titre d'insultes raciales et de coups et blessures.]

Le cas de la main courante est également intéressant, car il pose la question du statut de la parole publique lorsque celle-ci est adressée à un représentant judiciaire. La main courante s'inscrit dans un registre d'action relevant du préventif tant pour le justiciable que pour les services qui l'enregistrent (Ventre, Bauer, 2011). Déposer une main courante peut ainsi être préparatoire à la plainte puisqu'elle constitue une trace officielle du fait que, à une date et en un lieu donné, le justiciable a manifesté la volonté d'inscrire dans l'espace public une situation de vie difficile, l'engageant lui et ses proches. La main courante peut ainsi être mobilisée dans une procédure ultérieure - au pénal, au civil ou aux affaires familiales - pour attester de l'ancienneté du différend entre les parties et la récurrence des faits allégués. Mais la main courante peut aussi constituer un acte dilatoire puisque, en retenant son droit de porter plainte en cas d'infraction, le justiciable suspend l'enclenchement du processus de judiciarisation du différend. Il se donne ainsi, volontairement, ou sous les conseils des agents de la force publique, l'opportunité d'un temps de réflexion avant de procéder à une action en justice. Toutefois, il semble que la différence entre main courante et plainte en justice ne soit pas toujours bien identifiée par les justiciables, ce qui peut engager des défauts de compréhension par les plaignants, des temps de latence qui suivent l'acte de saisine de l'institution judiciaire.

Parce qu'il a eu recours aux institutions dans le cadre d'une dispute avec ses proches et qu'il porte à leur connaissance ce qui était considéré par l'un des 
disputant, par le groupe familial et, parfois, par l'environnement social plus large, comme relevant de l'entre soi, le plaignant occupe bien souvent la place inconfortable de « celui qui parle », du divulgateur, du délateur. C'est le cas par exemple pour les infidélités du conjoint qui sont régulièrement pointées dans les plaintes, mais aussi des histoires d'héritages et de filiation. Certaines choses sont, localement, bonnes à dire publiquement et d'autres moins ou pas du tout. Cette « économie de parler et de se taire », à laquelle Jean-François Laé et Isabelle Astier (2007) font référence dans leur étude sur les troubles de voisinage, s'applique également dans les situations de disputes interpersonnelles entre proches en Martinique. En tout état de cause, en passer par la plainte en justice, par le recours à l'institution judiciaire constitue localement un affront en soi. Cette dimension de la plainte s'enracine dans l'histoire locale du rapport aux institutions et au droit (Lantin, 2007). Ces freins à la plainte sont encore accentués par le jugement social qui peut être porté sur le plaignant selon que celui-ci est un homme ou une femme, en couple ou non, jeune ou âgé, selon que l'objet conflictuel est une insulte, une menace, une agression physique, etc., selon que sa relation à l'auteur des faits qu'il signale soit maritale, filiale, fraternelle, amicale, hiérarchique ${ }^{11}$. Le jugement porté sur la plainte et sur son auteur sera bienveillant ou stigmatisant : aller en justice pour un homme confronté à une agression verbale ou physique de la part d'un pair, c'est se montrer lâche (Bougerol, 2001) et s'exposer à être qualifié de makomé $e^{12}$ par exemple.

Selon la stratégie d'exposition de la plainte choisie, c'est-à-dire de description des faits et de catégorisation de ceux-ci, le plaignant s'expose et prend le risque de ce que je nomme ici un « retournement » de la plainte contre sa propre personne. En émettant une plainte ou en rendant publique une situation donnée, le plaignant encourt le risque de révéler des parts de sa vie et des zones de son intimité qui pourront ultérieurement favoriser un jugement négatif à son

11. Le terme « hiérarchique » recouvre ici les classifications locales que mobilisent, au quotidien aussi bien qu'en situation de conflit, les individus pour se qualifier et se distinguer les uns des autres. Ces classifications s'appuient sur un ensemble de paramètres faisant intervenir la combinaison de références aux grandes classes phénotypiques (métropolitains ou z'orey, békés, coolie, neg'bleu, chabin, mulâtre, etc.), aux classes socioéconomiques, à la nationalité et à l'origine. Isolément, aucune de ces références n'est suffisante pour recouvrir et expliquer les rapports sociaux aux Antilles françaises, qu'il s'agisse d'interactions en contexte familial, professionnel ou politique.

12. Ce terme peut être traduit par homosexuel, « femmelette » ou lâche selon les contextes interactionnels. Il peut être mobilisé à titre d'insulte autant qu'à titre de plaisanterie rituelle dans les groupes masculins d'interconnaissance. 
encontre. Le « retournement » de la plainte est ainsi le fait de prendre la parole pour dénoncer ce que l'on estime être une injustice ou une situation invivable et chercher un soutien de ses interlocuteurs et n'obtenir, à terme, qu'une mise en question de ses intentions, de sa crédibilité, de sa part de responsabilité, etc. Ainsi et en reprenant l'exemple donné par Elisabeth Stokoe et Alexa Hepburn (2005) sur les plaintes pour nuisances sonores entre voisins, l'exercice de présentation de soi est particulièrement difficile lorsqu'il existe un fort degré de proximité et d'interconnaissance entre les parties litigantes, car être un bon voisin (ou un bon « proche ») ce n'est pas être un dénonciateur, ce n'est pas non plus être un espion ou un voyeur, ce à quoi pourrait s'apparenter le fait d'avoir l'habitude d'observer ses voisins et le fait de connaître ce qui se passe chez eux. De fait et dans ces situations, se plaindre équivaut à énoncer un discours sur les activités intimes d'autrui, ce qui expose aussi le plaignant au jugement des récipiendaires de son discours et à un questionnement sur les procédés et les raisons pour lesquelles il sait ce qu'il avance.

\section{Des épreuves et des risques personnels et interactionnels}

À l'instar de ce que souligne Isabelle Coutant (2005, p. 47) pour les dépôts de plainte concernant des « délits de jeunesse », l'action en justice peut constituer un acte de résistance en soi : il peut s'agir de montrer à autrui que l'on ne se laisse pas faire et que l'on réagit, c'est utiliser la plainte pour « donner une leçon » ou encore s'emparer du droit et de la plainte pour « faire peur ». Devenir plaignant serait alors faire face à l'agresseur, occuper une place active, offensive ou moralisatrice.

Dans tous les cas, la personne confrontée à ce qui représente pour elle des offenses pour devenir plaignante doit faire part de son expérience à autrui et accepter de se livrer à un récit de soi (Fassin, 2000). La « peine à écrire ${ }^{13}$ et à dire la plainte se conjugue souvent dans les récits des plaignants à un sentiment d'urgence à exprimer publiquement ce qui est vécu pour pouvoir obtenir de l'aide. Les récits témoignent de la charge émotionnelle qui sous-tend le moment d'énonciation de la plainte : il est fait référence explicitement à différents registres d'émotion comme la peur, la honte, la colère, l'épuisement, l'indignation, etc. ; les silences et les hésitations rythment les prises de parole et, dans le face-à-face, les

13. Sophie FISHer, Michel de Fornel, 1998. 
gestes qui accompagnent le récit manifestent une inquiétude des narrateurs quant aux réactions de leur(s) interlocuteur(s) ${ }^{14}$.

MP, $I^{r e}$ séance, Mme Astier vs M. Belin (absent), violences avec arme, relations de voisinage

Mme A. (plaignante) : non j'ai, j'ai jamais été voir les femmes battues, mais une fois j'ai j'ai téléphoné comme ça et j'avais appelé, j'vais téléphoné pour en parler

Méd (médiatrice) : et vous avez laissé tomber...

Mme A. : non, j'ai pas réussi à...

Méd : vous avez pas réussi à parler, d'accord. [...]

Mme A. : c'est, c'est honteux d'en parler...

Méd : c'est honteux pour vous, c'est pas honteux d'en parler, il y a ici un service d'aide aux victimes aussi qui, euh, qui reçoit ici

[...]

Mme A. : oui, mais là ils vont... c'est, c'est que c'est tellement

honteux d'être la personne, tu parles à la personne et la personne va parler à d'autres...

Méd : [...] Vous êtes en droit de réclamer, c'est légitime de réclamer pour que les choses s'arrêtent et que ça ne recommence pas et que les personnes soient punies, d'accord ? C'est légitime de faire ça...

Dans l'extrait précédent, on voit que prendre la parole, dire ce que l'on vit, interpeller une institution et accéder à la plainte ne sont pas des décisions aisées à prendre. Se plaindre n'est pas un acte évident puisqu'il engage une exposition de soi et que celle-ci expose en retour le plaignant à l'appréciation qui pourra être faite par autrui de ses conduites, de son mode de vie, de son parcours biographique (ou certaines séquences de celui-ci), de sa personnalité et de sa responsabilité dans la situation désignée comme problématique.

$\mathrm{Si}$, en amont de la plainte, les plaignants affichent quelques hésitations comme le montre l'exemple précédent, en aval et une fois l'acte posé, ils doivent en assumer les

14. C'est tout particulièrement la quête visuelle des signes paraverbaux d'écoute, de compréhension et d'assentiment aux propos énoncés qui est repérable durant les rencontres de médiation et les entretiens ethnographiques : regard furtif en direction du médiateur ou de l'enquêteur, suspension de l'énoncé en attente d'une focalisation de l'interlocuteur sur le locuteur actif, ou encore, lors de certaines prises de parole, détournement du regard jusqu'à la clôture du récit. 
conséquences. Les récits de plaignants rétrospectifs à la plainte (médiation pénale, entretiens ethnographiques) signalent clairement que se plaindre a modifié la situation et les relations de celui-ci avec les proches incriminés, mais ces évolutions ne sont pas toujours celles escomptées par le plaignant. Dans certains cas, la réception de la convocation en justice par les personnes mises en cause entraîne une suspension des agressions verbales ou physiques, mais très souvent, les plaignants rapportent qu'il ne s'agit que d'une « pause » et non d'une véritable résolution du problème initialement signalé. Par ailleurs, ils sont nombreux à pointer que la judiciarisation du conflit suscite des formes de contre-attaques de la part de la personne ou du groupe familial mis en cause dans le litige (Bougerol, 2001). Ces contre-attaques peuvent être formulées dans le registre juridique, sous la forme d'une plainte réciproque ${ }^{15}$. Le plus souvent cependant le registre emprunté par l'accusé pour répliquer à l'action en justice s'inscrit dans la continuité de celui qui caractérisait les échanges antérieurs à la plainte (insultes, menaces, agressions physiques). Ce sont alors les modalités d'intensité, de densité et de portée des offenses qui varient : multiplication, aggravation, extension du périmètre concerné par les attaques qui se détachent de la cible singulière, le plaignant, pour atteindre ses proches, ses biens ou les activités qui constituent son moyen de subvenir à ses besoins.

Quand je lui ai annoncé que j'avais reçu l'autorisation judiciaire de quitter le toit : il a chargé son fusil de trois cartouches et a foncé sur moi. (LP2)

Je crois qu'il n'a pas bien compris ce qu'il a lu et ensuite signé. En effet c'est comme si vous n'avez rien dit, pour la bonne raison que c'est maintenant qu'il s'est levé contre moi [...] il m'a fait comme réponse un gros mot mal sain sur ma mère, que je ne peux exprimer comment vous le dire. Le [indication temporelle précise, jour et heure] ils se sont levés sur moi. (LP25)

Enfin et parfois, les contre-attaques se réaliseront de manière collective ou par le biais de tierces personnes, mobilisées par l'accusé à titre défensif et offensif à la fois (se protéger de l'action en justice et répliquer à celle-ci par l'usage de pratiques sorcellaires comme le quimbois par exemple). Les suites d'une action

15. On parle de « plainte réciproque » lorsque les parties en lice dans un litige ont mutuellement porté plainte en justice l'une contre l'autre, entraînant donc un recouvrement de leurs places actancielles : le plaignant est également le mis en cause et vice-et-versa. 
en justice signifient ainsi, dans les cas étudiés, un durcissement des positions des parties en lice : les qualités de conjoints, de membres d'une fratrie, de voisins, etc., s'estompent pour laisser place à celles de disputant et de partie litigante. Les échanges ordinaires, quand l'espace de vie continue d'être partagé, sont tous orientés et parasités par des glissements vers le « problème ». À ce propos, R. Emerson et S. Messinger (1977, p. 125) soulignent :

The trouble may become the direct focus of the relationship, generating a continuing dialogue in which what is wrong and what should be done about it are explored, possibilities elaborated, and options specified. In this way, a complaint not only organizes and consolidates the trouble, but also constitutes that trouble as a fully interpersonal matter.

\section{Les rhétoriques de la plainte en justice}

Certains des risques évoqués précédemment, et plus spécifiquement la disqualification judiciaire, sont anticipés par les plaignants qui cherchent à les déjouer en usant de certaines «ficelles » dans la construction de leur récit de plainte. Émettre une plainte c'est en effet se soumettre à l'exercice de mise en récit des événements qui sont à l'origine du différend ou qui le constituent. C'est aussi un exercice de portraiture des protagonistes du différend, à commencer par la personne du plaignant. Ces descriptions soutiennent plusieurs enjeux. D'une part, elles contribuent à susciter l'intérêt et l'empathie du récipiendaire de la plainte, d'autre part, elles doivent lui permettre de se faire une opinion sur la crédibilité de l'auteur de la plainte et sur la gravité des faits qui lui sont rapportés. Ces éléments, combinés avec ceux provenant des procès-verbaux et des indications des officiers de police judiciaire qui ont procédé aux auditions du plaignant et du contrevenant, permettent en dernière instance au procureur de la République de décider d'engager des poursuites contre l'auteur des faits signalés, d'orienter l'affaire vers la justice de proximité, ou encore de classer sans suite le dossier du plaignant.

\section{Occuper le terrain du droit}

En premier lieu, il s'agit pour le plaignant de construire sa plainte et de présenter sa cause en mobilisant des clés, notamment linguistiques et argumentatives, qui sont celles du droit. La connaissance, compréhension et maîtrise de ces « ficelles » dépend de nombreux éléments dont, entre autres, le parcours biographique, scolaire et professionnel du plaignant, ses précédentes expériences judiciaires, les appuis sociaux et économiques dont il peut disposer pour accompagner et guider 
son « chemin de plainte » et son parcours judiciaire ${ }^{16}$. La plainte en justice, écrite ou orale, est donc à plus d'un titre une performance de la part du plaignant : il emprunte régulièrement des tournures, des formules et des catégories juridiques pour se présenter, parler des faits, fonder ses arguments et, en somme, se battre sur le terrain du droit avec les armes de celui-ci ou, du moins, celles que le plaignant lui attribue. Certains courriers sont ainsi rédigés sur un registre impersonnel très marqué se rapprochant de l'écrit administratif (références à des numéros de procès-verbaux, à la côte de pièces juridiques, à des articles de loi, etc.), d'autres présentent un nombre élevé d'occurrences des termes « droit », « loi », « ordre public », etc. D'autres, encore, révèlent qu'un véritable travail d'enquête et de collationnement de justificatifs a été réalisé par le plaignant, ce dernier énumérant dans sa plainte tous les éléments qui sont en sa possession et qui corroborent ses assertions.

Ces pratiques montrent toutes que le travail de prise de parole sous la forme d'une plainte en justice requiert de la part des plaignants une préparation cognitive, matérielle, linguistique, de manière à anticiper les attentes normatives - avérées ou imaginées - du récipiendaire de la plainte (officier de police judiciaire, procureur de la République). Au-delà de cette dimension, cet exercice de formulation de la plainte en justice est sous-tendu par un travail de légitimation de la place qu'endosse le scripteur d'une plainte : celle du requérant dans une action en justice, celle de la victime, celle d'une personne qui est dans son bon droit.

Tout au long de leurs récits de plainte, les requérants témoignent de la légitimité de leur position - «Monsieur le Procureur de la République, oui je suis une victime » (LP12) - et de leur droit à obtenir les interventions qu'ils demandent, ainsi que les compensations qu'ils espèrent pour les griefs endurés. Ainsi les portraits qu'ils tracent d'eux-mêmes sont ceux de personnes dont les conduites répondent autant aux normes sociales que légales (sens civique, respect des lois et des contrats dont celui du mariage par exemple), dont les agissements dans le contexte conflictuel qui prévaut à leur plainte ont été empreints de tempérance, de patience et de raison : ils ont refusé le recours à la vengeance privée pour en appeler à la justice étatique, ils se sont gardés de répondre aux provocations et agressions, ils ont tapé à toutes les portes, ils ont prévenu le procureur à chaque nouvel incident, etc. Les plaignants décrivent leur parcours biographique et

16. On observe, au sein du corpus de lettres de plaintes analysé, une distribution inégale parmi les scripteurs des compétences et habitudes quant à l'acte d'écrire, et plus encore quant à celui de rédiger un écrit officiel (maladresse du tracé, ratures, maîtrise de l'orthographe et de la syntaxe, usage excessif de formules rhétoriques de politesse, etc.). 
l'histoire de leur conflit en se positionnant comme des personnes de «bonne moralité » présentant dans une situation critique une demande légitime et mesurée à l'institution judiciaire : une intervention pour assurer le rétablissement de l'ordre, de la justice et de conditions de vie «tranquilles ».

\section{Dramatiser}

Le caractère urgent et critique des situations que les plaignants vivent est mis en exergue par l'usage dans leurs récits de qualificatifs négatifs - invivable, insupportable, anormal, intolérable - par l'énumération de l'ensemble des actes et offenses subies et, enfin, en utilisant des formules qualifiées d' « extreme case formulation ${ }^{17}$. Ces dernières ont pour propriété d'intensifier leurs propos («il est toujours resté violent avec les enfants », LP27), mais aussi de susciter chez l'auditeur ou le lecteur une adhésion morale avec la perspective du narrateur et donc avec la lecture des faits qui lui est proposée.

L'un des ressorts sur lequel repose la dramatisation d'une affaire et l' interpellation du récipiendaire de la plainte est la présentation de facteurs aggravants les faits qui lui sont rapportés. À ce titre, la ligne d'argumentation majeure des plaignants est celle de leur vulnérabilité qui convoque le sens de l'éthique ou du devoir du récipiendaire de la plainte ainsi que sa capacité empathique et compassionnelle (Boltanski, 2011). Celui-ci est ainsi enjoint à écouter, à prendre en charge, à agir. La thématique de la vulnérabilité dans les récits de plaignants est utilisée d'une part pour dépeindre leur situation en temps ordinaire, ils signalent de multiples inégalités entre ce que sont leurs conditions de vie et celles de leur agresseur, puis ce motif est repris pour signaler que les actes posés par leur disputant accentuent encore les asymétries existantes. Sont ainsi mobilisés différents registres et tactiques de présentation de soi sous les aspects les plus « pitoyables $\gg^{18}$ : la vulnérabilité physique et psychologique, la vulnérabilité économique, des facteurs fragilisants comme l'isolement social ou l'éloignement de ses proches font ainsi partie des appuis argumentatifs des plaignants pour interpeller leurs interlocuteurs.

Dans cette logique d'exhibition de sa propre vulnérabilité, il y a mobilisation du corps, qu'il soit marqué par l'avancée du temps et le grand âge, ou qu'il soit atteint d'un handicap ou d'une maladie. Le registre de la santé est ainsi très régulièrement évoqué pour émouvoir l'interlocuteur et pour enchérir le statut de victime et d'individu requérant protection. Considérons le courrier suivant :

17. Anita Pomerantz, 1986 ; Derek Edwards, 2000.

18. Catherine BALLÉ, 1976. 
Monsieur le Procureur, ce n'est pas la première fois que je porte plainte contre ces individus méchants. Je suis à la retraite et je suis très malade. Je suis suivi par une infirmière qui se déplace tous les jours à mon domicile pour me soigner et vérifier mon état de santé. [...] Je continue à subir des menaces de mort et je vie dans la peur ; dans la crainte chaque jour et mon état de santé s'aggrave. [...] Maintenant, je suis à bout, je n'en peux plus, agissez vite avant qu'il ne soit trop tard. Je vous en supplie, je suis au bord de la dépression. Monsieur Eric Amber fait pression sur mon épouse qui est malade psychologiquement dont j'ai la charge. Il lui monte la tête contre moi dans ma maison. (LP7)

L'altération de la santé, la fragilité de la situation économique du plaignant ainsi que la présence des enfants lors des altercations peuvent être avancés comme des facteurs aggravant l'acte délictueux (s'attaquer à quelqu'un de démuni/fragile/ mineur) ainsi que ses conséquences directes et constituent donc des arguments supplémentaires pour justifier une rapide intervention des autorités. Ces éléments signifient également au récipiendaire de la plainte que les atteintes subies ont pour les solliciteurs de plus lourdes conséquences que pour d'autres et, élément aggravant, que l'accusé avait connaissance de ces fragilités au moment des faits signalés dans la plainte. C'est donc là, de la part des plaignants, autant l'intention malveillante de leur agresseur qui peut être soulignée qu'un appel à un traitement plus sévère de celui-ci ou à une compensation financière plus généreuse des dols subis.

\section{Criminaliser : l'altérité radicale de l'accusé}

Dans un certain nombre de leurs récits de conflit, les plaignants se livrent à un travail de portraiture de la personne mise en cause sous l'espèce du « méchant $»$, de la « brute », de l' « amoral » et, en somme, de l'impénitent. C'est la nature même de l'individu qui est mise en cause au-delà de la dénonciation de ses conduites. Qu'il s'agisse d'un père parlant de ses fils, « Monsieur le Procureur, ce n'est pas la première fois que je porte plainte contre ces individus méchants » (LP7), ou d'une épouse parlant de son conjoint, «Depuis un an les menaces sont plus violentes et de plus il met la pression utilisant toutes formes de méchancetés possibles pour [...] ma santé et me pousser au suicide, à une congestion où je pourrai laisser ma vie »(LP2). Les items méchant/méchanceté transparaissent régulièrement des descriptions des faits dénoncés, de la personnalité de leurs auteurs ou des mobiles 
de leurs actions ${ }^{19}$. L'énoncé suivant est porté par le plaignant dans le cadre d'une session de médiation pénale intervenant dans un contexte familial au sein d'une fratrie où des violences physiques et des agressions répétées se sont manifestées, entraînant une plainte de l'un des frères.

Plaignant : [...] il a fait marche arrière toute pour écraser les deux jambes de mon fils hein, il a deux visages, et lui-même j'ai jamais vu un bonhomme tout c'qu'y dit est mensonge, et tout c'qu'y dit c'est tout d'la méchanceté qu'il a dans son corps. Cet homme est un méchant et mauvais. Voyez ce que je veux dire. Alors avec moi y'a pas de médiation avec un machin comme ça (...) on peut pas négocier avec un un : bonhomme comme ça, non $[. .$.$] bonm-tala$ i tro méchant, i mové mem (ce bonhomme-là il est trop méchant, il est vraiment mauvais)

Les opposants vivent dans des maisonnées mitoyennes, érigées sur un espace indivis appartenant de longue date à la famille maternelle, ce qui entraîne de nombreuses querelles concernant le partage et l'usage du territoire, les manières de vivre de chaque groupe familial ainsi que la constante surveillance et appréciation mutuelle des conduites des membres de la fratrie envers leur mère (qui vit chez l'un d'eux). L'auteur des faits dénoncés est présenté comme relevant d'une nature autre que celle qui caractérise le plaignant (Ballé, 1976) : ses conduites seraient marquées par des passions excessives (consommation d'alcool, de stupéfiants), sa personnalité présenterait des carences affectives et émotionnelles (absence de sentiment paternel, désintérêt pour le foyer familial, incapacité à l'empathie, etc.), ses actes illustreraient son immoralité et son manque de valeurs (infidélité, plaisir à humilier et dominer autrui, mépris des promesses, des contrats, des lois et des institutions, etc.). En somme, le portrait du mis en cause que dresse le plaignant,

19. Dans les cas étudiés et du point de vue local, la « méchanceté » embrasse une série d'agissements et de comportements considérés comme irrespectueux envers autrui, les règles et valeurs de la vie en commun. Ces actes, initialement considérés comme triviaux, singuliers ou conjoncturels, sont progressivement ressentis et dénoncés comme violents sur le plan verbal, psychologique ou physique (insultes et menaces à répétition, port d'un couteau au ceinturon, menaces au fusil chargé, etc.) Ceux qui se plaignent interprètent ces actes comme intentionnels et comme des signes de projets plus vastes visant la destruction - symbolique ou réelle - de leur personne par leur opposant (menaces de mort, pressions sur autrui pour susciter son départ du foyer, l'abandon d'un bien ou d'une prérogative ou la modification d'un accord, etc.). Certains plaignants qualifient de « comble de la malignité » les actes égoïstes, ceux qui visent à les empêcher d'avancer dans la vie (« barrer la route ») ou à leur ôter leur tranquillité par simple jalousie. 
celui d'un être foncièrement et volontairement violent ou déviant, devient un ressort supplémentaire de dramatisation de la situation et de légitimation de la plainte.

Ce travail de portraiture établit une distance entre soi (le plaignant) et autrui (l'accusé) qu'accentuent les modes de dénomination du fauteur de trouble. Celui-ci est régulièrement désigné par son nom de famille même s'il s'agit du conjoint, du fils ou du père (de la conjointe, de la fille ou de la mère) et, plus souvent encore, on le nomme ou l'on s'adresse à lui avec le titre «monsieur/madame » précédé d'un démonstratif (《ce », « cette »), voire suivi du nom de famille. La locution «ce monsieur-là » - dérivée du langage parlé et du créole boug-la/misyé-tala - dénote, en contexte conflictuel et lorsque l'interlocuteur dont on parle est présent, une mise à distance voire du mépris pour la personne dont il est question.

\section{Collectiviser le problème ou monter en généralité}

Les modalités de construction des récits de plainte, de présentation de soi comme requérant et justiciable dans le corpus étudié ne répondent pas systématiquement aux exigences de « dé-singularisation », de « distance » et de « détachement » qui constituent la « condition de félicité des actes de parole qui portent la critique dans l'espace public $\gg{ }^{20}$. Cette maxime de la parole critique dans l'espace public a été énoncée par de nombreux auteurs ayant notamment travaillé sur les actes - et surtout sur les écrits - de dénonciation (Boltanski et al., 1984) ou de réclamation (Pinto, 1989 ; Borzeix et al., 1995 ; Cardon, 1995 ; Branca-Rosoff, 1997). Cette maxime est à l'œuvre dans certaines lettres de plainte adressées au procureur de notre corpus, mais, le plus souvent, les scripteurs jouent simultanément sur plusieurs registres argumentatifs et imbriquent au sein d'une même lettre des registres contraires (désingulariser et personnaliser par exemple). De plus, lorsque plusieurs lettres sont écrites par un même plaignant, on observe que cette maxime est applicable pour l'une ou l'autre de ces lettres, mais pas forcément à l'œuvre au sein de chacune.

On observe que, dans tout ou partie de leur lettre de plainte et de leurs récits ultérieurs sur leur action en justice, les plaignants s'appuient sur deux grands ordres de références légitimant d'une part le fait même d'avoir porté plainte et justifiant d'autre part leurs attentes en matière d'intervention judiciaire. Le premier mode de légitimation de la plainte s'appuie sur une référence aux principes et valeurs au nom desquels les plaignants déclarent agir. Ils font référence à une

20. Dominique Cardon, Jean-Philippe Heurtin, Cyril Lemieux, 1995, p. 9. 
norme légale et à une conception du monde ou de l'ordre social organisée par le droit. Le conflit est alors présenté comme dérangeant ce monde et le plaignant occupe, en s'adressant au procureur, la position de celui qui dénonce des irrégularités, des infractions et de celui qui requiert une restauration de l'ordre légal. Les plaignants convoquent ainsi les notions d' « État de droit », de « sécurité publique », « d'ordre » (public). Ils font également référence à la « confiance » qu'ils placent dans les institutions et en particulier dans l'institution judiciaire et dans la personne à laquelle ils s'adressent dans la présente situation de rédaction d'une lettre de plainte, c'est-à-dire au procureur de la République. Par ailleurs, ils ne manquent pas de rappeler, particulièrement en ouverture et en clôture de leurs courriers, que l'institution judiciaire et ses représentants se doivent d'être les garants du respect de la loi et ont, à ce titre, des responsabilités dont entre autres celle de satisfaire aux requêtes en justice que leur soumettent les plaignants. L'attente du plaignant est donc la mise en action, par les représentants de l'État auxquels il s'adresse, des moyens juridiques qu'ils ont à leur disposition : procès, sanctions, etc.

J'aimerais en toutes liberté, égalité, fraternité, sous réserve de mes droits passés, présents et futurs, que dans cette affaire les auteurs [noms] ne reconnaissent plus leur tort et cherche à me déstabiliser afin d'obtenir que ma plainte soit considérée comme nulle et qu'il ne soit donné aucune suite devant la médiation. [...] Je vous demande donc de faire procéder que cette affaire soit envoyée devant un tribunal pour faire valoir ce qui est de droit. Monsieur le Procureur de la République, j'ai confiance en la justice. (LP12)

Étant donné que nous sommes dans un pays de droit, je vous sollicite afin de faire aboutir ma plainte, et que justice me soit rendue. (LP11)

Le second mode de légitimation de l'action en justice s'appuie, lui, sur la référence à des personnes au nom desquelles l'attention et l'intervention des récipiendaires de la plainte sont requises. Il peut s'agir d'un autrui généralisé (les citoyens, tous les Martiniquais, la collectivité, la société), d'un autrui identifié et personnalisé vivant ou décédé (ma famille, mes enfants, la mémoire d'un parent décédé), ou encore d'entités non humaines (l'ordre public, la loi, etc.). Dans notre corpus, constitué de plaintes concernant des différends entre proches, les tiers les plus concernés dans les plaintes qui mobilisent l'argument « agir au nom d'un tiers » sont les enfants et, en seconde position les parents proches. Le recours au procureur au nom d'une personne présentée comme vulnérable - un enfant, un 
parent âgé, malade ou à charge - cherche aussi à susciter l'empathie du magistrat et à motiver une action de type compassionnel de sa part. Le plaignant présente son recours au droit comme un moyen pour lui de défendre une personne fragile et une manière de signaler aux autorités une situation qui lui semble inconvenable. La protection de l'enfant est un principe largement mobilisé dans les lettres de plainte « au nom d'un tiers » légitime.

Elle est en $6^{\mathrm{e}}$, a-t-elle le droit d'avoir une telle vie pour un homme qui refuse de quitter les lieux sous prétexte qu'il ne peut pas payer un loyer, ou qui cherche à nous détruire avant son départ. (LP9)

Monsieur le Procureur de la République Française, les problèmes du couple ne m'intéressent pas autant que les problèmes pschychologiques [sic] et sûrement sociaux qu'ils provoquent déjà chez mon petit-fils. (LP13)

Dans les deux cas, celui qui se plaint au nom d'autrui comme celui qui se plaint au nom de principes et de valeurs se distancie de la figure du bénéficiaire de la plainte : s'il pose une action en justice ce n'est pas pour lui, mais pour autrui ou au nom d'un principe et de valeurs élevées. Ce n'est donc ni par l'appât du gain, ni pour porter préjudice à son adversaire, ni pour acquérir sur lui un quelconque pouvoir, ni encore pour la simple satisfaction d'être reconnu pour être celui qui a raison. Si le plaignant n'est plus le bénéficiaire alors sa plainte est « dépouillée » d'une partie de sa charge subjective et émotionnelle (Boltanski, 2011).

Un dernier moyen pour le plaignant de légitimer sa plainte est de construire la situation qu'il signale comme un cas «typique » participant d'une série de cas similaires déjà reconnus dans l'espace public comme des problèmes méritant l'intervention des forces publiques. Ainsi les plaignants peuvent, dans leurs récits, faire référence à des faits divers ayant connu des suites dramatiques ${ }^{21}$. En comparant leur cas à celui de ces personnes, ils espèrent retenir l'attention du récipiendaire de leur plainte et susciter une intervention rapide. En s'appuyant sur ces faits-divers, les plaignants avancent que de tels « drames » peuvent (leur) arriver et ils enjoignent l'institution judiciaire à agir pour que le nombre de victimes de ce type de situations et de violences ne s'élève pas plus et pour que la/ leur « tranquillité » soit garantie ou restaurée.

21. Lors de notre terrain, plusieurs faits divers de violences conjugales (par exemple, l'immolation par son ex-conjoint d'une femme en pleine rue) avaient suscité des articles dans le quotidien local et des manifestations publiques (marches blanches). 


\section{Conclusion}

Comme nous avons pu le montrer, la plainte en justice est une prise de parole par laquelle un problème est porté à la connaissance de l'institution judiciaire qui va se charger, dans certains cas et sous certaines conditions, d'officialiser celui-ci sous la forme du litige. En recourant à la plainte en justice, son auteur fait reconnaître publiquement ses expériences et son interprétation d'une situation donnée afin que soit ouverte une affaire judiciaire. Au cours de ce chemin de plainte en justice le plaignant est amené pour s'assurer que sa plainte emportera l'attention et l'adhésion de son récipiendaire à passer sous silence certains aspects de l'histoire du conflit et de la situation qui le préoccupe. Cette économie du dire et du taire dans le processus de production de la plainte soulève la question de l'efficacité de la parole publique comme mode de résolution des problèmes : qu'est-il fait après la plainte et dans le traitement judiciaire du litige, de ce qui a été laissé en suspens, effacé, tu ? L'analyse des médiations pénales nous permet en partie de répondre à cette question puisque, dans cette enceinte, qui s'est ouverte à la faveur d'une plainte en justice, la parole du plaignant est confrontée à celle des proches désignés comme accusés lors de la plainte. Ceux-ci viennent réinscrire dans le débat sur le problème, sur sa genèse et sur les remédiations possibles à celui-ci, ce qui a été éludé par la plainte en justice. Mais, cette fois-ci les échanges se passeront dans le huis clos de la médiation et ne seront pas fixés par écrit si ce n'est, exceptionnellement, par l'ethnologue.

\section{Bibliographie}

BALlÉ Catherine, 1976, la Menace : un langage de violence, Paris : CNRS Éditions.

Boltanski Luc, Darré Yann, Schiltz Marie-Ange, 1984, « la Dénonciation », Actes de la Recherche en Sciences Sociales, n 51, p. 3-40.

Boltanski Luc, 2011, [1990], l'Amour et la Justice comme compétences, Paris : Gallimard.

Borzeix Annie, Fisher Sophie, De Fornel Michel, Lacoste Michèle, 1995, «les Lettres de réclamation », in Claude QUIN (dir.), l'Administration de l'équipement et ses usagers, Paris : la Documentation Française, p. 71-106. 
Bougerol Christiane, 1997, une Ethnographie des conflits aux Antilles : jalousie, commérages, sorcellerie, Paris : PUF.

Bougerol Christiane, 1999, « la Sorcellerie aux Antilles : interactions et malheurs », Socio-Anthropologie, no 5, p. 57-67.

Bougerol Christiane, 2001, « Mots de plainte et Mots de menace : lettres au procureur de la République en Guadeloupe », l'Homme, vol. 4, n 160, p. 117-135.

Bourdieu Pierre, 1982, « les Rites comme des actes d'institution », Actes de la Recherche en Sciences Sociales, $\mathrm{n}^{\circ}$ 43, p. 58-63.

BrancA-Rosoff Sonia, 1997, « les Lettres de réclamation adressées au service de la redevance », Langage et Société, n ${ }^{\circ} 81$, p. 69-86.

Cardon Dominique, 1995, « Comment se faire entendre ? La prise de parole des auditeurs de RTL », Politix, vol. 8, n³1, p. 145-186.

Cardon Dominique, Heurtin Jean-Philippe, Lemieux Cyril, 1995, « Parler en public $\gg$, Politix, vol. 8, n 31, p. 5-19.

Coutant Isabelle, 2005, Délits de jeunesse : la justice face aux quartiers, Paris : la Découverte.

DE Fornel Michel, 1988, « Sociopragmatique de la conversation : production, réception et séquentialisation des récits de plainte », Cabiers du français des années quatre-vingt, $\mathrm{n}^{\circ}$ 3, p. 165-188.

EDWARDs Derek, 2000, "Extreme Case Formulations. Softeners, Investment, and Doing NonLiteral”, Research on Language and Social Interaction, vol. 33, $\mathrm{n}^{\circ} 4$, p. 347-373.

Emerson Robert M., Messinger Sheldon L., 1977, "The Micro-Politics of Trouble", Social Problems, n 25, p. 121-135.

FAssin Didier, 2000, « la Supplique : stratégies rhétoriques et constructions identitaires dans les demandes d'aide d'urgence », Annales Histoire, Sciences Sociales, vol. 55, n 5, p. 955-981. 
FISHer Sophie, De Fornel Michel, 1998, la Volonté d'écrire : étude des pratiques d'écriture dans un atelier d'écriture, rapport de recherche du Ministère de la Culture, Mission du patrimoine ethnologique.

Foli Olivia, 2006, Plaintes, Normes et Intégration : le cas d'une organisation bureaucratique, thèse de sociologie, université Paris Dauphine.

González Martínez Esther, 2003, l'Audition de comparution immédiate : l'organisation des échanges langagiers, thèse de sociologie, université de Lausanne - EHESS.

LAÉ Jean-François, Murard Numa, 1995, les Récits du malheur, Paris : Descartes $\&$ Cie.

LaÉ Jean-François, Astier Isabelle, 2007, « le Trouble du voisinage, le droit de dire ou de se taire? », Rhizome, $\mathrm{n}^{\circ} 29$, p. 10-13.

Lantin Mickaële, 2007, la Justice de proximité en Martinique : médiateurs et conciliateurs. Pistes pour repenser la place du tiers dans les conflits, rapport de recherche du Ministère de la Culture, Mission du patrimoine ethnologique.

Pinto Louis, 1989, « Du pépin au litige en consommation : une étude du sens juridique ordinaire », Actes de la Recherche en Sciences Sociales, n ${ }^{\circ}$ 76-77, p. 65-81.

Pomerantz Anita, 1986, "Extreme Case formulation. A way of legitimizing claims", Human Studies, n 9, p. 219-229.

Retieres Jean-Noël, 2001, «En retard pour l'aide d'urgence : analyse de courriers de demandeurs $\gg$, Revue française des affaires sociales, $\mathrm{n}^{\circ}$ 1-1, p. 167-183.

Stokoe Elisabeth, Hepburn Alexa, 2005, "You can hear a lot through the walls, Noise formulations in neighbour complaints”, Discourse \& Society, vol. 16, $\mathrm{n}^{\circ} 5$, p. 647-673.

Ventre André-Michel, Bauer Alain, 2011, « Éditorial », Bulletin statistique de l'observatoire national de la délinquance et des réponses pénales, Éditions de l'INHESJ. 
170 Paroles publiques, paroles confidentielles $-n^{\circ} 77-78$

Résumé : Cet article analyse les modalités langagières et les processus par lesquels des individus s'adressent à l'institution judiciaire afin d'obtenir une reconnaissance et une prise en charge de ce qu'ils estiment être des situations insupportables. Dans le cours d'un conflit interpersonnel, lorsqu'un des participants décide de porter plainte en justice, cet acte constitue une prise de parole publique déplaçant ce qui était jusqu'alors un conflit d'ordre privé vers une arène judiciaire. L'examen des lettres de plainte adressées au procureur de la République de la Martinique et l'analyse des échanges qui se sont tenus, ultérieurement, dans le cadre des rencontres de médiation pénale permettent d'identifier d'une part les évènements ayant contribué à une telle prise de parole, mais aussi les moyens rhétoriques mobilisés par les plaignants pour faire de leur cause une affaire judiciaire et pour se constituer en plaignant légitime.

Mots clés : Martinique, droit, parole, plainte en justice, conflit, médiation, interlocution

Abstract:This article analyzes the ways of speaking and processes by which individuals turn to the judicial system in order to obtain recognition and support for what they consider to be unbearable situations. When, in the course of an interpersonal conflict, one of the participants decides to file a complaint in court, this action moves what is initially a private conflict to a public and judicial arena. Examination of complaint letters that are sent to the prosecutor of Martinique, and the analysis of exchanges that take place within the framework of criminal mediation meetings, allow the identification of events that contribute to the initial "voicing act", and of the rhetorical resources that are mobilized by the complainants to turn their case into a court case and present themselves as legitimate complainants.

Keywords: Martinique, Law, Speech, Legal Complaint, Conflict, Alternative Dispute Resolution, Interlocution 